\title{
A THREE-STEP EXAMINATION IN DIFFERENTIATING PERIPHERAL FROM CENTRAL VERTIGO
}

\author{
Irina Orban¹, Laura Dumitrescu' ${ }^{1}$ Bogdan O. Popescu ${ }^{1,2,3}$, Radu Tanasescu ${ }^{1,2}$ \\ ${ }^{1}$ Department of Neurology, Colentina Hospital, Bucharest, Romania \\ ${ }^{2}$ Department of Neurology, Neurosurgery and Psychiatry, University of Medicine and Pharmacy \\ Bucharest, Romania \\ ${ }^{3}$ Laboratory of Molecular Medicine and Neuroscience, "Victor Babes" National Institute of \\ Pathology, Bucharest, Romania
}

\section{INTRODUCTION}

Dizziness is the reason of $10 \%$ of emergency department visits (1). The asssessment of acute balance problems and vertigo in the A\&E setting or on the ward is often a challenge to the young neurologist. The ideal test to differentiate central from peripheral vertigo should be straightforward, cheap and with both good sensitivity and specificity. What best than a clinical series of tests easy to be performed at the bedside and able to replace brain MRI, when assessing a vestibular syndrome in the acute phase?

\section{CASE PRESENTATION}

We report the case of a 65-year old woman with multiple cardiovascular risk factors (hypertension, hyperlipidemia, insulino-necessitant diabetes mellitus) and a medical history of coronary artery disease (myocardial infarction with angioplasty and stenting 6 months prior to the admission), who presented with a acute positional vertigo with sudden onset a few hours prior to the admission.

At examination on the ward she complained of persistent vertigo with significant head motion intolerance and gait unsteadiness, accompanied by nausea, vomiting and chest pain. She was also having occipital headache without meningeal signs, non-responsive to usual analgetics and denied any hearing problems.
The neurological examination revealed horizontal nystagmus on lateral gaze to the right, left tonic deviation of the arms and a positive Romberg test with left laterodeviation. No other focal neurologic deficits were noted. The CT scan performed at admission was normal.

In brief, this patient with a significant risk for vascular disease was having an acute vestibular syndrome. The problem was differentiating between a peripheral and a central vertigo, the latter being often the result of a posterior circulation stroke. In our case, the neurological examination rather points towards a peripheral cause of the persistent vertigo (vestibular maneouvres elicited a tendency of laterodeviation to the left), although the patient's medical history suggests that central vertigo could also be considered. Based upon these findings, how certain are we of the peripheral or central origin of the neurological symptomatology?

\section{DISCUSSION}

Dizziness or vertigo accounts for approximately 2.6 million emergency department visits in the USA annually; acute vestibular syndrome is diagnosed in about 150000 cases (1). Of these, posterior circulation infarctions account for $25 \%$ or more of the cases (1). The diagnosis must be made with extreme caution since stroke (in particular the lateral medullary, lateral pontine, and inferior cere- 
bellar stroke) may very closely mimic an acute peripheral vestibulopathy (1). Vertebral artery dissection is one of the causes of posterior circulation stroke in young adults and can mimic an acute peripheral vestibular syndrome (1). Another fact that increases the diagnostic difficulty is that cerebellar infarctions are rarely associated with typical neurological signs, in about half of them these signs being completely absent (1).

Neuroradiological tests are useful in assesing an acute vestibular syndrome. A study suggests that the diagnostic yield for head CT is rather low, while MRI plays an important role in the evaluation of vertigo, in particular in appropriately selected cases (2). However, the utility of the MRI DWI in the diagnosis of posterior circulation infarctions is limited, especially in the first 24 hours after symptom onset, the frequency of false-negative diffusion weighted MRI being increased in the case of hyperacute ischemic stroke (3).

Taking into account these facts, recent data suggest a series of bed-side manoeuvres useful in differentiating between peripheral and central vertigo. The HINTS examination represents the evaluation of three oculomotor abnormalities, namely horizontal head impulse, the evaluation of nystagmus and the alternate eye cover testing or the test of skew.

The horizontal head impulse implies that the examiner rapidly rotates the head of the subject, while the vision of the latter is fixed on a static target. A negative head impulse test involves an intact vestibulo-ocular reflex and implies that the vision of the subject remains on the target, while the head is rotated. On the other hand, a positive head impulse involves an impaired vestibulo-ocular reflex and implies that the subject is unable to mantain the vision fixed on the target and needs to perform a corrective saccade after the rotation of the head in order to fix the target. In patients with vertigo, a positive horizontal head impulse suggests a peripheral vestibular syndrome, while a negative result is seen in central localization. However, there have been cases with abnormal head impulse test who actually have a central vestibular syndrome, namely lateral pontine infarcts (1). In these cases, the other two tests from the HINTS examination could help in the differential diagnosis. All in all, the horizontal head impulse test is considered the most consistent predictor of stroke in the setting of acute vestibular syndrome (1). Also, it has been shown that while a negative result is a strong predictor for a central localization, a positive result is rather a weak predictor for a peripheral localization (1).
The second component of the HINTS examination implies the evaluation of nystagmus, since the characteristics of the nystagmus suggests a certain diagnosis. In peripheral vestibular syndrome, the nystagmus is horizontal, increases when the vision is in the direction of the fast-phase, while the direction of the slow-phase indicates the affected vestibular system and beats in only one direction. On the other hand, a vertical or rotational nystagmus and a nystagmus that changes direction on eccentric gaze is a strong indicator of a central localization. Sometimes, a nystagmus can be predominantly horizontal even in central vestibular syndromes, the element differentiating the peripheral from the central cause being the change in the direction of the nystagmus. (1)

The alternate cover test is the third component of the examination and implies a vertical ocular misalignement due to right-left imbalance in otolith function. It consists in covering the eyes alternatively, while the subject is asked to fix his gaze on a static target and evaluating the position of the suddenly uncovered eye. A positive result implies that the suddenly uncovered eye has to change its position in order to fix the target and is indicative of a central lesion. The skew deviation test was found to have a low sensitivity and a high specificity for a central topography and was considered very useful especially in the setting of a false negative horizontal head impulse test (1).

In conclusion, in a patient with an acute vestibular syndrome a peripheral lesion would associate a positive horizontal head impulse, a direction fixed horizontal nystagmus and a negative skew test, while a central lesion would result in a negative horizontal head impulse, a direction-changing horizontal, rotational or vertical nystagmus and a positive skew test.

In the case of our patient, all three manoeuvres were normal and the ENT consultant confirmed the peripheral vertigo using vestibulo-posturography. Brain MRI was normal.

The HINTS evaluation is a valuable examination tool that helps in the diagnosis of acute vestibular syndrome. Importantly, a normal HINTS is a better tool to rule out a central vestibular syndrome compared with diffusion-weighted MRI performed in the first 48 hours after symptom onset (1). Moreover, finding an abnormality suggesting a central lesion in one of the three components is more sensitive than finding together all the traditional neurologic signs of posterior circulation infarction (1). HINTS evaluation was compared with the $\mathrm{ABCD} 2$ risk stratification score (ABCD2 stands 
for age, blood pressure, clinical features, duration of symptoms and diabetes) and revealed that the former outperforms significantly the latter in terms of association with an established stroke (4). HINTS is a straightforward and helpful series of tests when assesing acute balance problems.

\section{REFERENCES}

1. Kattah J.C., Talkad A.V., Wang D.Z., Hsieh Y.H., Newman-Toker D.E. HINTS to diagnose stroke in the acute vestibular syndrome: three-step bedside oculomotor examination more sensitive than early MRI diffusion-weighted imaging. Stroke. 2009 Nov; 40(11):3504-10.

2. Lawhn-Heath C., Buckle C., Christoforidis G., Straus C. Utility of head $\mathrm{CT}$ in the evaluation of vertigo/dizziness in the emergency department.; Epub 2012 Sep 2; 20(1):45-9

3. Morita S., Suzuki M., lizuka K. False-negative diffusion-weighted MRI in acute cerebellar stroke.; Epub 2011 Feb 16; 38(5):577-82

4. Newman-Toker D.E., Kerber K.A., Hsieh Y.H., Pula J.H., Omron R., Saber Tehrani A.S., Mantokoudis G., Hanley D.F., Zee D.S., Kattah J.C. HINTS outperforms ABCD2 to screen for stroke in acute continuous vertigo and dizziness.; Acad Emerg Med. 2013 Oct; 20(10):986-96 